\title{
LINEAR FEEDBACK CONTROL OF NONLINEAR SYSTEMS WITH A DOMINATING CONSERVATIVE QUADRATIC TERM
}

\author{
ANIL BOSE, ALAN COVER, and JAMES RENEKE
}

Department of Mathematical Sciences

Clemson University

Clemson, SC 29634-1907

(Received January 27, 1993 and in revised form January 27, 1997)

\section{Abstract}

Conditions are investigated for systems of the form $\boldsymbol{M} \boldsymbol{x}^{\prime}=N+\boldsymbol{A x}+\boldsymbol{f}(\boldsymbol{x})$, where $\boldsymbol{f}$ is quadratic, which yield a point dissipative system. Application of the conditions are made to the problem of existence of linear feedback controls $\boldsymbol{u}=K \boldsymbol{x}$ for systems of the form

$$
M \boldsymbol{x}^{\prime}=N+A \boldsymbol{x}+\boldsymbol{f}(\boldsymbol{x})+\boldsymbol{B u}
$$

which force the system to be point dissipative. The basic results have extensions to more general classes of systems.

\section{Introduction}

We are concerned with a class of nonlinear $n$-dimensional systems of the form

$$
M \boldsymbol{x}^{\prime}=N+A \boldsymbol{x}+\boldsymbol{f}(\boldsymbol{x}),
$$

where $M$ is a positive definite matrix and the nonlinear term $\boldsymbol{f}(\boldsymbol{x})$ is quadratic of the form

$$
f(x)=\left[\begin{array}{c}
x^{T} C_{1} x \\
\vdots \\
x^{T} C_{1} x
\end{array}\right]
$$

The $n \times n$ matrices $\left\{C_{i}\right\}$ are symmetric with the orthogonality property $\boldsymbol{x}^{T} \boldsymbol{f}(\boldsymbol{x})=0$ for all $\boldsymbol{x}$. Functions $f$ with the orthogonality property are said to be conservative. We start with an investigation of relations between the $n \times n$ matrix $A$ and the function $f$ that are sufficient to insure that the system is point dissipative, i.e., which guarantee the existence of a bounded region in $R^{n}$ which every trajectory of the system eventually enters and remains within. Such conditions would imply the system has a bounded attractor. As an example of a point dissipative system of the form given above, we cite the well known Lorenz system in Lorenz [15] and Sparrow [17], see also Guckenheimer [10] and Wiggins [18], $\boldsymbol{x}^{\prime}=A \boldsymbol{x}+\boldsymbol{f}(\boldsymbol{x})$, where

$$
\begin{gathered}
A=\left[\begin{array}{ccc}
-a & a & 0 \\
\gamma & -1 & 0 \\
0 & 0 & -b
\end{array}\right], a>0, \gamma>0, b>0 \\
\boldsymbol{f}(\boldsymbol{x})=\left[\begin{array}{c}
0 \\
-x_{1} x_{3} \\
x_{1} x_{2}
\end{array}\right], \boldsymbol{x}=\left[\begin{array}{l}
x_{1} \\
x_{2} \\
x_{3}
\end{array}\right]
\end{gathered}
$$


Note that $\boldsymbol{f}$ has the orthogonality property. Furthermore, if $\boldsymbol{u}$ is a nontrivial member of the zero set of $\boldsymbol{f}$, i.e., $\boldsymbol{u}=\left(u_{1}, 0,0\right)^{T}$ or $\boldsymbol{u}=\left(0, u_{2}, u_{3}\right)^{T}$ then $\boldsymbol{u}^{T} A \boldsymbol{u}<0$. Rotations preserve both of these properties.

When $n=2$ or 3 , a sufficient condition for the system to be point dissipative, see Bose $[6,1]$ is that $\boldsymbol{u}^{T} A \boldsymbol{u}<0$ for nontrivial $\boldsymbol{u}$ in the zero set of $\boldsymbol{f}$. The conjecture that this condition is sufficient for $n$-dimensional systems is unresolved except for special cases, i.e., systems where the zero set of $f$ satisfies additional conditions. Theorem 1 extends the $n$-dimensional result in Bose [1].

Since linear feedback does not disturb the zero set of $\boldsymbol{f}$, our feeling is that linear control minimally modifies the structure of the uncontrolled system. Our goal is to produce a linear feedback so the controlled system either has the origin as an asymptotic stable point or is point dissipative. No use is made of nonlinear feedback.

The extensions in Section 4 relax the conditions on $f(x)$, i.e., $f(x)$ need not be conservative or quadratic. Some of the results in this paper were announced in Bose [3] without proof.

\section{Basic Results}

It can be shown, see Bose [1] that for quadratic $f(x)$ with the orthogonality property the set $Z_{\boldsymbol{f}}=\{\boldsymbol{x} \mid \boldsymbol{f}(\boldsymbol{x})=\mathbf{0}\}$ contains at least a 1-dimensional subspace of $R^{n}$. For each vector $\boldsymbol{a}^{T}=$ $\left(a_{1}^{\prime}, a_{2}, \ldots, a_{n}\right)$, we define the $n \times n$ matrix $C(\boldsymbol{\alpha})$ as follows:

$$
C(\alpha)=\sum_{i=1}^{n} \alpha_{i} C_{i}-\frac{A+A^{T}}{2}
$$

Our first result is the following lemma.

Lemma 1 If there exists an $\alpha$ so that the matrix $C(\alpha)$ is positive definite then the system $\boldsymbol{x}^{\prime}=$ $A \boldsymbol{x}+\boldsymbol{f}(\boldsymbol{x})$ is point dissipative.

This lemma is proven by a standard argument using the Lyapunov function

$$
V(x)=\frac{1}{2}(x-\alpha)^{T} M(x-\alpha)
$$

For large $\|x\|$ the time derivative of $V(x(t))$ is dominated by $-x^{T}(t) C(\alpha) \mathrm{x}(t)$, see Bose [1]. Vectors $\boldsymbol{\alpha}$ for which $C(\boldsymbol{\alpha})$ is positive definite are said to be admissible for the system $\boldsymbol{x}^{\prime}=A \boldsymbol{x}+\boldsymbol{f}(\boldsymbol{x})$. A condition on the matrix $A$ and $\boldsymbol{f}(\boldsymbol{x})$ which guarantees the existence of admissible $\alpha$ 's is the topic of our next result. The condition $\boldsymbol{u}^{T} A \boldsymbol{u}<0$. for all nontrivial $u$ in $Z_{f}$ is necessary for the existence of an admissible $\alpha$. We have shown in Bose [1] the condition is sufficient when $n$ is 2 or 3 . We have also shown in Bose $[2,4]$ that this condition is sufficient when $Z_{f}$ is an $(n-1)$-dimensional subspace of $R^{n}$. The next result weakens this equality to inclusion. The method of proof presented here contains some new ideas not found in Bose [4].

Theorem 1 Consider the system $M x^{\prime}=A x+f(x)$, where $f$ is conservative. If $Z_{f}$ contains an $(n-1)$-dimensional subspace of $R^{n}$ then $\boldsymbol{u}^{T} A u<0$ for all nontrivial $\boldsymbol{u}$ in $Z_{f}$ if and only if there is an admissible $\alpha$ for the system.

We start by considering the conservative quadratic function $f(x)=\left[\begin{array}{c}x^{T} C_{1} x \\ \vdots \\ x^{T} C_{n} x\end{array}\right]$. If we define $x Q y=\left[\begin{array}{c}x^{T} C_{1} y \\ \vdots \\ x^{T} C_{n} y\end{array}\right]$ then $Q$ is a bilinear function and $f(x)=x Q x$. The vector space generated or spanned by $\boldsymbol{u}_{1}, \boldsymbol{u}_{2}, \ldots, \boldsymbol{u}_{k}$ is denoted by $S\left(\boldsymbol{u}_{1}, \boldsymbol{u}_{2}, \ldots, \boldsymbol{u}_{k}\right)$. 
Lemma 2 If $S\left(\boldsymbol{u}_{1}, \boldsymbol{u}_{2}, \ldots, \boldsymbol{u}_{k}\right) \subset Z_{\boldsymbol{f}}, \boldsymbol{u}_{1}, \boldsymbol{u}_{2}, \ldots, \boldsymbol{u}_{k}$ are linearly independent, and $\boldsymbol{u}^{T} A \boldsymbol{u}<\mathbf{0}$ for all nontrivial $u$ in $Z_{f}$ then the matrix $B=\left[-x^{T} A y\right]_{k \times k}$, is positive definite.

Proof. Let $\boldsymbol{x}$ be an element of $S\left(\boldsymbol{u}_{1}, \boldsymbol{u}_{2}, \ldots, \boldsymbol{u}_{k}\right)$.. Then $\boldsymbol{x}=\sum_{t=0}^{k} c_{1} \boldsymbol{u}_{i}$ and, by hypothesis, $\boldsymbol{u}^{T} A \boldsymbol{u}<0$ for all nontrivial $\boldsymbol{u}$ in $Z_{\boldsymbol{f}}$. Therefore

$$
0<-\boldsymbol{x}^{T} A \boldsymbol{x}-\left(\sum_{i=1}^{k}\left(c_{i} \boldsymbol{u}_{i}\right)^{T} A\left(c_{i} \boldsymbol{u}_{i}\right)\right)=\left(c_{1}, \ldots, c_{k}\right) B\left[\begin{array}{c}
c_{1} \\
\vdots \\
c_{k}
\end{array}\right]
$$

implies $B$ is positive definite.

Lemma 3 The subspace $S\left(\boldsymbol{u}_{1}, \boldsymbol{u}_{2}, \ldots, \boldsymbol{u}_{k}\right)$, is contained in $Z_{\boldsymbol{f}}$, if and only if $\boldsymbol{u}_{i} Q \boldsymbol{u}_{j}=0$ for all $i, j=1,2, \ldots, k$.

Proof. Suppose that $S\left(\boldsymbol{u}_{1}, \boldsymbol{u}_{2}, \ldots, \boldsymbol{u}_{k}\right) \subseteq Z_{\boldsymbol{f}}$. Then $S\left(\boldsymbol{u}_{i}, \boldsymbol{u}_{j}\right) \subseteq Z_{\boldsymbol{f}}$ and $\boldsymbol{u}_{i}, \boldsymbol{u}_{j}$, and $\boldsymbol{u}_{i}+\boldsymbol{u}_{j} \in$ $S\left(\boldsymbol{u}_{\imath}, \boldsymbol{u}_{\jmath}\right)$. Therefore

$$
0=f\left(u_{i}+u_{\jmath}\right)=f\left(u_{i}\right)+2 u_{i} Q u_{\jmath}+f\left(u_{\jmath}\right)=2 u_{\imath} Q u_{\jmath}
$$

Conversely suppose that $\boldsymbol{u}_{i} Q \boldsymbol{u}_{j}=0$ for all $i, j=1,2, \ldots, k$. Let $\boldsymbol{x} \in S\left(\boldsymbol{u}_{1}, \boldsymbol{u}_{2}, \ldots, \boldsymbol{u}_{k}\right)$ so that $\boldsymbol{x}=\sum_{\mathfrak{r}=1}^{k} c_{\mathrm{t}} \boldsymbol{u}_{\boldsymbol{i}}$. Now

$$
\boldsymbol{f}(\boldsymbol{x})=\boldsymbol{f}\left(\sum_{i=1}^{k} c_{i} \boldsymbol{u}_{i}\right)=\sum_{i=1}^{k} \sum_{j=1}^{k} c_{i} c_{j} \boldsymbol{u}_{i} Q u_{j}
$$

which implies that $S\left(\boldsymbol{u}_{1}, \boldsymbol{u}_{2}, \ldots, \boldsymbol{u}_{k}\right) \subseteq Z_{\boldsymbol{f}}$.

Lemma 4 When $Z_{f}$ contains an $(n-1)$-dimensional subspace then there is a basis $\boldsymbol{u}_{1}, \boldsymbol{u}_{2}, \ldots, \boldsymbol{u}_{n}$ of $R^{n}$ such that $Z_{\boldsymbol{f}}=S\left(\boldsymbol{u}_{1}, \boldsymbol{u}_{2}, \ldots, \boldsymbol{u}_{n-1}\right)$ or $Z_{\boldsymbol{f}}=S\left(\boldsymbol{u}_{1}, \boldsymbol{u}_{2}, \ldots, \boldsymbol{u}_{n-1}\right) \cup S\left(\boldsymbol{u}_{k+1}, \boldsymbol{u}_{k+2}, \ldots, \boldsymbol{u}_{n}\right)$.

Proof. When $Z_{f}$ contains an $(n-1)$-dimensional subspace then $Z_{f}$ is either an $(n-1)$-dimensional subspace or $Z_{f}$ is the union of an (n-1)-dimensional subspace $H$ and another subspace $K$ Bose [5]. When $K$ is nontrivial, let $\boldsymbol{u}_{k}, \boldsymbol{u}_{k+2}, \ldots, \boldsymbol{u}_{n-1}$ be a basis of the intersection of $H$ and $K$. Then there is a basis $\boldsymbol{u}_{1}, \boldsymbol{u}_{2}, \ldots, \boldsymbol{u}_{n}$ of $R^{n}$ such that $\boldsymbol{u}_{1}, \boldsymbol{u}_{2}, \ldots, \boldsymbol{u}_{n-1}$ is a basis of $H$ and $\boldsymbol{u}_{k+1}, \boldsymbol{u}_{k+2}, \ldots, \boldsymbol{u}_{n}$ is a basis of $K$.

Proof of Theorem 1. Suppose that $S\left(\boldsymbol{u}_{1}, \boldsymbol{u}_{2}, \ldots, \boldsymbol{u}_{n-1}\right) \subseteq Z_{\boldsymbol{f}}$. Let $\boldsymbol{u}_{1}, \boldsymbol{u}_{2}, \ldots, \boldsymbol{u}_{n}$ be any basis for $R^{n}$. Then for any $\boldsymbol{x}$ in $R^{n}$ we have $\boldsymbol{x}=\sum_{i=1}^{n} p_{i} \boldsymbol{u}_{i}$ and

$$
\boldsymbol{x}^{T} C(\boldsymbol{\alpha}) \boldsymbol{x}=\boldsymbol{p}^{T} \hat{C}(\boldsymbol{\alpha}) \boldsymbol{p}=
$$

$$
\boldsymbol{p}_{T}\left[\begin{array}{cccc}
-\boldsymbol{u}_{1}^{T} A \boldsymbol{u}_{1} & \ldots & -\boldsymbol{u}_{1}^{T} A \boldsymbol{u}_{n-1} & \boldsymbol{\alpha}^{T} \boldsymbol{u}_{1} Q \boldsymbol{u}_{n}-\boldsymbol{u}_{1}^{T} A u_{n} \\
-\boldsymbol{u}_{2}^{T} A \boldsymbol{u}_{1} & \ldots & -\boldsymbol{u}_{2}^{T} A u_{n-1} & \boldsymbol{\alpha}^{T} \boldsymbol{u}_{2} Q \boldsymbol{u}_{n}-\boldsymbol{u}_{2}^{T} A \boldsymbol{u}_{n} \\
\vdots & \vdots \vdots & \vdots & \vdots \\
-\boldsymbol{u}_{n-1}^{T} A \boldsymbol{u}_{1} & \ldots & -\boldsymbol{u}_{n-1}^{T} A u_{n-1} & \boldsymbol{\alpha}^{T} \boldsymbol{u}_{n-1} Q u_{n}-\boldsymbol{u}_{n-1}^{T} A \boldsymbol{u}_{n} \\
\boldsymbol{\alpha}^{T} \boldsymbol{u}_{1} Q \boldsymbol{u}_{n}-\boldsymbol{u}_{1}^{T} A u_{n} & \ldots & \boldsymbol{\alpha}^{T} \boldsymbol{u}_{n-1} Q \boldsymbol{u}_{n}-\boldsymbol{u}_{n-1}^{T} A \boldsymbol{u}_{n} & \boldsymbol{\alpha}^{T} \boldsymbol{u}_{n} Q \boldsymbol{u}_{n}-\boldsymbol{u}_{n}^{T} A \boldsymbol{u}_{n}
\end{array}\right] \boldsymbol{p}
$$

where $\boldsymbol{p}^{T}=\left(p_{1}, p_{2}, \ldots, p_{n}\right)$. This last quadratic function of $\boldsymbol{p}$ can be written as

$$
\boldsymbol{p}^{T}\left[\begin{array}{cc}
B & b \\
b^{T} & \alpha^{T} u_{n} Q u_{n}-u_{n}^{T} A u_{n}
\end{array}\right] p
$$


where

$$
\boldsymbol{b}^{T}=\left(\boldsymbol{\alpha}^{T} \boldsymbol{u}_{1} Q \boldsymbol{u}_{n}-\boldsymbol{u}_{1}^{T} A \boldsymbol{u}_{n}, \ldots, \boldsymbol{\alpha}^{T} \boldsymbol{u}_{n-1} Q \boldsymbol{u}_{n}-\boldsymbol{u}_{n-1}^{T} A \boldsymbol{u}_{n}\right)
$$

and $B$ is the $(n-1) \times(n-1)$ matrix of Lemma 2 and is therefore positive definite. Hence $C(\boldsymbol{\alpha})$ is positive definite for some $\boldsymbol{\alpha}$ if and only if $\hat{C}(\boldsymbol{\alpha})$ is positive definite for some $\boldsymbol{\alpha}$. In order to show that $\hat{C}(\boldsymbol{\alpha})$ is positive definite we need only show further that $\operatorname{det}(\hat{C}(\boldsymbol{\alpha}))>0$.

Case 1. Suppose that $S\left(\boldsymbol{u}_{1}, \boldsymbol{u}_{2}, \ldots, \boldsymbol{u}_{n-1}\right)=Z_{\boldsymbol{f}}$. A different proof appears in [4]. In this case $\boldsymbol{u}_{n} Q \boldsymbol{u}_{n} \neq 0$ and $\boldsymbol{u}_{n} Q \boldsymbol{u}_{n}$ can not belong to $S\left(\boldsymbol{u}_{1} Q \boldsymbol{u}_{n}, \boldsymbol{u}_{2} Q \boldsymbol{u}_{n}, \ldots, \boldsymbol{u}_{n-1} Q \boldsymbol{u}_{n}\right)$. For $\boldsymbol{u}_{n} Q \boldsymbol{u}_{n}=$ $\sum_{t=1}^{n-1} d_{\imath} u_{\imath} Q u_{n}$ implies that

$$
f\left(u_{n}-\sum_{i=1}^{n-1} \frac{d_{i}}{2} u_{\imath}\right)=u_{n} Q u_{n}-\sum_{i=1}^{n-1} d_{i} u_{i} Q u_{n}=0
$$

which in turn implies that $\boldsymbol{u}_{n}-\sum_{\mathfrak{t}=1}^{n-1} \frac{d_{n}}{2} \boldsymbol{u}_{i}$ is in $Z_{f}=S\left(\boldsymbol{u}_{1}, \boldsymbol{u}_{2}, \ldots, \boldsymbol{u}_{n-1}\right)$ contradicting our hypothesis.

Let $\operatorname{dim} S\left(\boldsymbol{u}_{1} Q \boldsymbol{u}_{n}, \ldots, \boldsymbol{u}_{n-1} Q \boldsymbol{u}_{n}\right)=k, k \leq n-1$. Without loss of generality, suppose that $\boldsymbol{u}_{1} Q \boldsymbol{u}_{n}, \ldots, \boldsymbol{u}_{k} Q \boldsymbol{u}_{n}$ are linearly independent. Then $S\left(\boldsymbol{u}_{1} Q \boldsymbol{u}_{n}, \ldots, \boldsymbol{u}_{n-1} Q \boldsymbol{u}_{n}\right)$

$=S\left(\boldsymbol{u}_{1} Q \boldsymbol{u}_{n}, \ldots, \boldsymbol{u}_{k} Q \boldsymbol{u}_{n}\right)$ and

$$
\boldsymbol{u}_{k+\jmath} Q \boldsymbol{u}_{n}=\sum_{i=1}^{k} d_{j i} \boldsymbol{u}_{\imath} Q \boldsymbol{u}_{n}, \quad j=1, \ldots, n-k-1
$$

Hence $S\left(\boldsymbol{u}_{1} Q \boldsymbol{u}_{n}, \ldots, \boldsymbol{u}_{k} Q \boldsymbol{u}_{n}, \boldsymbol{u}_{n} Q \boldsymbol{u}_{n}\right)$ has dimension $k+1$ and so, for each $\tau>0$, there exists a vector $\boldsymbol{\alpha}_{\tau}$ such that $\boldsymbol{\alpha}_{\tau}^{T} \boldsymbol{u}_{i} Q \boldsymbol{u}_{n}-\boldsymbol{u}_{\boldsymbol{\imath}}^{T} A \boldsymbol{u}_{n}=0, \quad i=1, \ldots, k$, and $\boldsymbol{\alpha}_{\tau}^{T} \boldsymbol{u}_{n} Q \boldsymbol{u}_{n}-\boldsymbol{u}_{n}^{T} A \boldsymbol{u}_{n}=\boldsymbol{\tau}$. For such an $\boldsymbol{\alpha}_{\tau}$, the last row of $\hat{C}\left(\boldsymbol{\alpha}_{\tau}\right)$ becomes $\left(0, \ldots, 0, b_{k+1}, \ldots, b_{n-1}, \tau\right)$, where $b_{k+\jmath}$, for $j=$ $1, \ldots, n-k-1$, does not depend on $\boldsymbol{\alpha}_{\tau}$ but rather on $\boldsymbol{u}_{1}^{T} A \boldsymbol{u}_{n}, \ldots, \boldsymbol{u}_{n-1}^{T} A \boldsymbol{u}_{n}$ and $d_{\jmath \imath}, \quad i=1, \ldots, k$. Let $b_{T}=\left(0, \ldots, 0, b_{k+1}, \ldots, b_{n-1}\right)$. Now $\operatorname{det}\left(\hat{C}\left(\boldsymbol{\alpha}_{\tau}\right)\right)=\left(\boldsymbol{\tau}-\boldsymbol{b}^{T} B^{-1} \boldsymbol{b}\right) \operatorname{det} B$ see Horn [11]. We can choose $\tau$ large enough to make $\tau>b^{T} B^{-1} b$ and, for such an $\tau, \hat{C}\left(\alpha_{\tau}\right)$ is positive definite. Hence $C\left(\boldsymbol{\alpha}_{\tau}\right)$ is positive definite, i.e., $\boldsymbol{\alpha}_{\tau}$ is admissible.

Case 2. Suppose $Z_{f}$ is the union of an $(n-1)$-dimensional subspace $H$ and another subspace $K$ neither of which is trivial. Let $\boldsymbol{u}_{1}, \boldsymbol{u}_{2}, \ldots, \boldsymbol{u}_{n}$ be the basis given in Lemma 4, in particular $H=S\left(\boldsymbol{u}_{1}, \ldots, \boldsymbol{u}_{n}\right)$ and $K=S\left(\boldsymbol{u}_{k+1}, \ldots, \boldsymbol{u}_{n}\right)$. As in Case 1, the matrix $\hat{C}(\boldsymbol{\alpha})$ in this case is of the form

where

$$
\boldsymbol{p}^{T}\left[\begin{array}{cc}
B & b \\
b^{T} & -u_{n}^{T} A u_{n}
\end{array}\right] p
$$

$$
\boldsymbol{b}^{T}=\left(\boldsymbol{\alpha}^{T} \boldsymbol{u}_{1} Q u_{n}-\boldsymbol{u}_{1}^{T} A u_{n}, \ldots, \alpha^{T} u_{k} Q u_{n}-\boldsymbol{u}_{k}^{T} A u_{n},-u_{k+1}^{T} A u_{n}, \ldots,-u_{n-1}^{T} A u_{n}\right)
$$

and $B$ is the same as in Case 1. Also the $(n-k) \times(n-k)$ matrix

$$
D=\left[\begin{array}{cccc}
-\boldsymbol{u}_{k+1}^{T} A \boldsymbol{u}_{k+1} & -\boldsymbol{u}_{k+1}^{T} A \boldsymbol{u}_{k+2} & \ldots & -\boldsymbol{u}_{k+1}^{T} A \boldsymbol{u}_{n} \\
-\boldsymbol{u}_{k+1}^{T} A \boldsymbol{u}_{k+2} & -\boldsymbol{u}_{k+2}^{T} A \boldsymbol{u}_{k+2} & \ldots & -\boldsymbol{u}_{k+2}^{T} A \boldsymbol{u}_{n} \\
\vdots & \vdots & \vdots \vdots & \vdots \\
-\boldsymbol{u}_{k+1}^{T} A \boldsymbol{u}_{n} & -\boldsymbol{u}_{k+2}^{T} A \boldsymbol{u}_{n} & \ldots & -\boldsymbol{u}_{n}^{T} A \boldsymbol{u}_{n}
\end{array}\right]
$$

is positive definite by hypothesis and Lemma 2 . We can write

$$
D=\left[\begin{array}{cc}
B_{3} & a \\
a^{T} & -u_{n}^{T} A u_{n}
\end{array}\right]
$$

where $a^{T}=\left(-u_{k+1}^{T} A u_{n}, \ldots,-u_{n-1}^{T} A u_{n}\right)$ and the $(n-k-1) \times(n-k-1)$ matrix $B_{3}$ is positive definite. Also $\operatorname{det}(D)=\operatorname{det}\left(-u_{n}^{T} A u_{n}-a^{T} B_{3}^{T} a\right) \operatorname{det}\left(B_{3}\right)>0$. Since $\operatorname{det}\left(B_{3}\right)>0$, this implies that

$$
\left(-u_{n}^{T} A u_{n}-a^{T} B_{3}^{T} a\right) \operatorname{det}\left(B_{3}\right)>0
$$


The vectors $\boldsymbol{u}_{1} Q \boldsymbol{u}_{n}, \ldots, \boldsymbol{u}_{k} Q \boldsymbol{u}_{n}$ are linearly independent. Otherwise, $\sum_{i=1}^{k} p_{i} \boldsymbol{u}_{1} Q \boldsymbol{u}_{n}=0$ with $\boldsymbol{p}^{T}=\left(p_{1}, \ldots, p_{k}\right) \neq 0$ implies that

$$
\boldsymbol{f}\left(\boldsymbol{u}_{n}+\frac{1}{2} \sum_{i=1}^{k} p_{i} \boldsymbol{u}_{i}\right)=\sum_{i=1}^{k} p_{i} u_{\imath} Q u_{n}=0
$$

This in turn implies that $\boldsymbol{u}_{n}+\frac{1}{2} \sum_{s=1}^{k} p_{i} \boldsymbol{u}_{\mathfrak{i}} \in Z_{\boldsymbol{f}}$ and so $\boldsymbol{u}_{n} \in H$, a contradiction. Hence for each $k$ vector $\left(w_{1}, \ldots, w_{k}\right)=\boldsymbol{w}^{T}$, there exists an $\boldsymbol{\alpha}(\boldsymbol{w})$ so that $\boldsymbol{\alpha}(\boldsymbol{w})^{T} \boldsymbol{u}_{\imath} Q \boldsymbol{u}_{n}-\boldsymbol{u}_{i}^{T} A \boldsymbol{u}_{n}=w_{i}, i=1, \ldots, k$. Let $\boldsymbol{y}^{T}=\left(w_{1}, \ldots, w_{k},-\boldsymbol{u}_{k+1}^{T} A \boldsymbol{u}_{n}, \ldots,-\boldsymbol{u}_{n-1}^{T} A \boldsymbol{u}_{n}\right)=\left(\boldsymbol{w}^{T}, \boldsymbol{a}^{T}\right)$. Then $\operatorname{det}\left(\hat{C}(\boldsymbol{\alpha}(\boldsymbol{w}))=\left(-\boldsymbol{u}_{n}^{T} A \boldsymbol{u}_{n}-\right.\right.$ $\left.\boldsymbol{y}^{T} B^{-1} \boldsymbol{y}\right) \operatorname{det}(B)$. In block form $B=\left[\begin{array}{cc}B_{1} & B_{2} \\ B_{2}^{T} & B_{3}\end{array}\right]$ and $B^{-1}=\left[\begin{array}{cc}E_{1} & E_{2} \\ E_{2}^{T} & E_{3}\end{array}\right]$ where $B_{1}$ and $E_{1}$ are $k \times k$ positive definite matrices and $B_{3}$ and $E_{3}$ are positive definite $(n-1-k) \times(n-1-k)$ matrices. Note that $B_{3}$ was defined as a submatrix of $D$.

In order to show that $\boldsymbol{w}_{0}=-E_{1}^{-1} E_{2} a$ is a choice of $\boldsymbol{w}$ so that $\operatorname{det}(\hat{C}(\boldsymbol{\alpha}(\boldsymbol{w}))>0$ we would like to show that $E_{3}-E_{2}^{T} E_{1}^{-1} E_{2}=B_{3}^{-1}$. In block form

$$
\left[\begin{array}{ll}
I & 0 \\
0 & I
\end{array}\right]=\left[\begin{array}{cc}
B_{1} E_{1}+B_{2} E_{2}^{T} & B_{1} E_{2}+B_{1} E_{3} \\
B_{2}^{T} E_{1}+B_{3} E_{2}^{T} & B_{2}^{T} E_{2}+B_{3} E_{3}
\end{array}\right]
$$

This gives us two equations in which we are interested, $B_{2}^{T} E_{2}+B_{3} E_{3}=I$ and $B_{2}^{T} E_{1}+B_{3} E_{2}^{T}=0$. The first implies that $E_{3}=B_{3}^{-1} B_{2}^{T} E_{2}$ and the second implies $E_{2}^{T} E_{1}^{-1}=-B_{3}^{-1} B_{2}^{T}$. Hence

$$
E_{3}-E_{2}^{T} E_{1} E_{2}=B_{3}^{-1}-\left(B_{3}^{-1} B_{2}^{T}+E_{2}^{T} E_{1}^{-1}\right) E_{2}=B_{3}^{-1}-\left(B_{3}^{-1} B_{2}^{T}+B_{3}^{-1} B_{2}^{T}\right) E_{2}=B_{3}^{-1} .
$$

Moreover, for $y_{0}=\left(w_{0}, a^{T}\right)$,

$$
\boldsymbol{y}_{0}^{T} B^{-1} y_{0}=w_{0}^{T} E_{1} w_{0}+2 w_{0}^{T} E_{2} a+a^{T} E_{3} a=a^{T}\left(E_{3}-E_{2}^{T} E_{1}^{-1} E_{2}\right) a=a^{T} B_{3}^{-1} a .
$$

Since by $(2.1)-u_{n}^{T} A u_{n}-a^{T} B_{3}^{-1} a>0$,

$$
\operatorname{det}\left(\hat{C}\left(\boldsymbol{\alpha}\left(\boldsymbol{w}_{0}\right)\right)=\left(-\boldsymbol{u}_{n}^{T} A \boldsymbol{u}_{n}-\boldsymbol{y}_{0}^{T} B_{3}^{-1} \boldsymbol{y}_{0}\right) \operatorname{det}(B)=\left(-\boldsymbol{u}_{n}^{T} A \boldsymbol{u}_{n}-\boldsymbol{a}^{T} B_{3}^{-1} \boldsymbol{a}\right) \operatorname{det} B>0 .\right.
$$

Therefore $C\left(\boldsymbol{\alpha}\left(\boldsymbol{w}_{0}\right)\right)$ is positive definite, i.e., $\boldsymbol{\alpha}\left(\boldsymbol{w}_{0}\right)$ is admissible, and Theorem 1 follows from Lemma 1.

\section{Control Results}

For our next result we consider nonlinear control systems of the form

$$
M \boldsymbol{x}^{\prime}=N+A \boldsymbol{x}+\boldsymbol{f}(\boldsymbol{x})+B \boldsymbol{u},
$$

where $B$ is an $n \times m$ matrix and $u$ is an $m$-vector. Two types of general behavior are investigated:

1. Existence of a linear feedback control $\boldsymbol{u}=K \boldsymbol{x}$ so that the system $M \boldsymbol{x}^{\prime}=N+$ $(A+B K) \boldsymbol{x}+\boldsymbol{f}(\boldsymbol{x})+B \boldsymbol{u}$ has the origin as a global asymptotic stable point.

2. Existence of a linear feedback control $\boldsymbol{u}=K \boldsymbol{x}$ so that the system $M \boldsymbol{x}^{\prime}=N+$ $(A+B K) \boldsymbol{x}+\boldsymbol{f}(\boldsymbol{x})+B \boldsymbol{u}$ is point dissipative.

Let $S(B)$ be the column space of $B$ and $B^{\perp}$ be the orthogonal complement of $S(B)$.

Lemma 5 If $\mathcal{M}$ is a nonempty closed subset of $\left\{x \mid x \in B^{\perp}\right.$ and $\|x\|=1$ and $\left.x^{T} A x<0\right\}$ and $\mathcal{N}$ is a nonempty closed subset of $\{\boldsymbol{x} \mid \boldsymbol{x} \in S(B)$ and $\|\boldsymbol{x}\|=1\}$ then there is a negative number $\tau$ such that $\boldsymbol{v}^{T}\left(A+\tau B B^{T}\right) v<0$ for $v \in S(\mathcal{M}) \oplus S(\mathcal{N})$. 
Proof. $\mathcal{M} \times \mathcal{N}$ is compact in $R^{n} \times R^{n}$. Since the function

$$
\phi(\beta, \nu)=\frac{\left(\beta^{T} A \beta\right)\left(\nu^{T} A \nu\right)-\left(\beta^{T} \nu\right)^{2}}{\left(\beta^{T} A \beta\right)\left\|B^{T} v\right\|^{2}}
$$

is continuous on $\mathcal{M} \times \mathcal{N}$, there exists $\left(\boldsymbol{\beta}_{0}, \nu_{0}\right) \in \mathcal{M} \times \mathcal{N}$, such that $\phi(\boldsymbol{\beta}, \boldsymbol{\nu}) \leq \phi\left(\boldsymbol{\beta}_{0}, \boldsymbol{\nu}_{0}\right)$ for all $(\boldsymbol{\beta}, \nu) \in \mathcal{M} \times \mathcal{N}$. Choose a negative number $\tau$ such that $\phi\left(\boldsymbol{\beta}_{0}, \nu_{0}\right)+\tau<0$. For such an $\tau, \phi(\beta, \nu)+\tau \leq \phi\left(\boldsymbol{\beta}_{0}, \nu_{0}\right)+\tau<0$, for all $(\boldsymbol{\beta}, \boldsymbol{\nu}) \in \mathcal{M} \times \mathcal{N}$. That is

$$
\phi(\boldsymbol{\beta}, \boldsymbol{\nu})=\frac{\left(\boldsymbol{\beta}^{T} A \boldsymbol{\beta}\right)\left(\boldsymbol{\nu}^{T} A \nu\right)-\left(\boldsymbol{\beta}^{T} \boldsymbol{\nu}\right)^{2}}{\left(\boldsymbol{\beta}^{T} A \boldsymbol{\beta}\right)\left\|B^{T} \boldsymbol{\nu}\right\|^{2}}+\tau<0
$$

for all $(\boldsymbol{\beta}, \boldsymbol{\nu}) \in \mathcal{M} \times \mathcal{N}$. Since $\boldsymbol{\beta}^{T} A \boldsymbol{\beta}<0$, by hypothesis, we have $\left(\boldsymbol{\beta}^{T} A \boldsymbol{\beta}\right)\left(\boldsymbol{\nu}^{T} A \boldsymbol{\nu}\right)-\tau\left(\boldsymbol{\beta}^{T} A \boldsymbol{\beta}\right)$. $\left\|B^{T} \nu\right\|^{2}-\left(\beta^{T} A \nu\right)^{2}>0$ for all $(\boldsymbol{\beta}, \nu) \in \mathcal{M} \times \mathcal{N}$.

If $\boldsymbol{x}=a \boldsymbol{\beta}+b \boldsymbol{\nu}$, where $(\boldsymbol{\beta}, \boldsymbol{\nu}) \in \mathcal{M} \times \mathcal{N}$ and $a$ and $b$ are scalars, then

$$
\begin{aligned}
\boldsymbol{x}^{T}\left(A+\tau B B^{T}\right) \boldsymbol{x} & =(a \boldsymbol{\beta}+b \boldsymbol{\nu})^{T}\left(A+\tau B B^{T}\right)(a \boldsymbol{\beta}+b \boldsymbol{\nu}) \\
& =a^{2} \boldsymbol{\beta}^{T} A \boldsymbol{\beta}+2 a b \boldsymbol{\beta}^{T} A \nu+b^{2}\left(\boldsymbol{\nu}^{T} A \nu+\tau\left\|B^{T} \nu\right\|^{2}\right) \\
& =[a, b]\left[\begin{array}{cc}
\boldsymbol{\beta}^{T} A \boldsymbol{\beta} & \boldsymbol{\beta}^{T} A \nu \\
\boldsymbol{\beta}^{T} A \nu & \nu^{T} A \nu+\tau\left\|B^{T} \boldsymbol{\nu}\right\|^{2}
\end{array}\right]\left[\begin{array}{l}
a \\
b
\end{array}\right]=[a, b] L\left[\begin{array}{l}
a \\
b
\end{array}\right]
\end{aligned}
$$

Now consider the matrix $-L$. Since $-\boldsymbol{\beta}^{T} A \boldsymbol{\beta}>0$ and the $\operatorname{det}(-L)=\left(\boldsymbol{\beta}^{T} A \boldsymbol{\beta}\right)\left(\boldsymbol{\nu}^{T} A \boldsymbol{\nu}\right)+\tau\left\|B^{T} \boldsymbol{\nu}\right\|^{2}$ $\boldsymbol{\beta}^{T} A \boldsymbol{\beta}-\left(\boldsymbol{\beta}^{T} A \nu\right)^{2}>0$ for all $(\boldsymbol{\beta}, \boldsymbol{\nu}) \in \mathcal{M} \times \mathcal{N},-L$ is positive definite. Hence $\boldsymbol{x}^{T}\left(A+\tau B B^{T}\right)^{T} \boldsymbol{x}<0$.

Theorem 2 There exists a matrix $K$ such that the system $\mathbf{M x}^{\prime}=(A+B K) \boldsymbol{x}+\boldsymbol{f}(\boldsymbol{x})$ has the origin as a global asymptotic stable point if $\boldsymbol{u}^{T} A \boldsymbol{u}<0$ for each nontrivial vector $\boldsymbol{u}$ which is orthogonal to the column space of $B$.

Proof. Note that $R^{n}=B^{\perp} \oplus S(B)$. Let $\operatorname{dim} S(B)=k, 1 \leq k \leq n$, then $\operatorname{dim} S^{\perp}=n-k$. If $k=n$ then the result is immediate. Assume $k<n$. Let $\mathcal{M}=\left\{\boldsymbol{x} \mid \boldsymbol{x} \in B^{\perp},\|\boldsymbol{x}\|=1\right\}$ and $\mathcal{N}=\{\boldsymbol{x} \mid \boldsymbol{x} \in S(B),\|\boldsymbol{x}\|=1\}$. By Lemma 5 there is a negative number $\tau$ such that $x^{T}\left(A+\tau B B^{T}\right) x<0$ for all nontrival $x \in R^{n}$. Let $K=\tau B^{T}$.

Consider the Lyapunov function $V(x)=\frac{1}{2} x^{T} M x$, then

$$
\dot{V}(\boldsymbol{x})=\boldsymbol{x}^{T} M\left[M^{-1}\left\{\left(A+\tau B B^{T}\right) \boldsymbol{x}+\boldsymbol{f}(\boldsymbol{x})\right\}\right]=\boldsymbol{x}^{T}\left(A+\tau B B^{T}\right) \boldsymbol{x}<0
$$

where $M \boldsymbol{x}^{\prime}=(A+B K) \boldsymbol{x}+\boldsymbol{f}(\boldsymbol{x})$ and $x(t) \neq 0$. Hence by Lyapunov's Theorem in LaSalle [14] or Brauer [7] the system $M \boldsymbol{x}^{\prime}=(A+B K) \boldsymbol{x}+\boldsymbol{f}(\boldsymbol{x})$ has the origin as a global asymptotic stable point. Compare with standard results for linear feedback control of linear systems in Cruz [9] or Russell [16].

Example for Theorem 2. Consider the control system $\boldsymbol{x}^{\prime}=A \boldsymbol{x}+f(\boldsymbol{x})+B \boldsymbol{u}$, where

$$
A=\left[\begin{array}{ccc}
3 & 1 & -4 \\
2 & -1 & 0 \\
-4 & 0 & 1
\end{array}\right], \quad \boldsymbol{f}(\boldsymbol{x})=\left[\begin{array}{c}
0 \\
-x z \\
x y
\end{array}\right] \text { and } B=\left[\begin{array}{c}
1 \\
0 \\
-1
\end{array}\right]
$$

Here

$$
B^{\perp}=S\left\{\left[\begin{array}{l}
0 \\
1 \\
0
\end{array}\right],\left[\begin{array}{l}
1 \\
0 \\
1
\end{array}\right]\right\} \text { and } Z_{f}=S\left\{\left[\begin{array}{l}
0 \\
1 \\
0
\end{array}\right],\left[\begin{array}{l}
0 \\
0 \\
1
\end{array}\right]\right\} \cup S\left\{\left[\begin{array}{l}
1 \\
0 \\
0
\end{array}\right]\right\}
$$


Note that $\boldsymbol{u} \in B^{\perp}$ and $\boldsymbol{u} \neq 0$ implies $\boldsymbol{u}^{T} A \boldsymbol{u}<0$. Take $K=\tau B^{T}$. Then

$$
A+B K=A+\tau B B^{T}=\left[\begin{array}{ccc}
3+\tau & 1 & -4-\tau \\
2 & -1 & 0 \\
-4-\tau & 0 & 1+\tau
\end{array}\right]
$$

The Hermitian part of $A+\tau B B^{T}$ is given by

$$
\left[\begin{array}{ccc}
3+\tau & \frac{3}{2} & -4-\tau \\
\frac{3}{2} & -1 & 0 \\
-4-\tau & 0 & 1+\tau
\end{array}\right]
$$

which is negative definite when $\tau=-7$. Hence the system $x^{\prime}=\left(A-7 B B^{T}\right) x+f(x)$ is globally asymptotically stable. When $\tau=-4$ the system is point dissipative since $x^{T}\left(A-4 B B^{T}\right) x<0$ for all nontrival zeros of $\boldsymbol{f}$.

Five trajectories of the controlled system

$$
\frac{d x}{d t}=\left(\left[\begin{array}{ccc}
3 & 1 & -4 \\
2 & -1 & 0 \\
-4 & 0 & 1
\end{array}\right]+\tau\left[\begin{array}{c}
1 \\
0 \\
-1
\end{array}\right]\left[\begin{array}{lll}
1 & 0 & -1
\end{array}\right]\right) x+\left[\begin{array}{c}
0 \\
-x z \\
x y
\end{array}\right]
$$

are shown in Figure 1. The initial point of all of the trajectories is $(-10,10,10)$. One trajectory is when there is no control, that is, $\tau=0$. This is the long trajectory that is at $(-329,-4,7)$ when $t=1.2$. This trajectory seems to be moving right along at that time. The other trajectories are when the control parameter $\tau=-4,-5,-6$, and -7 . These trajectories seem to reach a limit point of the controlled system, namely, $(-1.7,-1.7,1.0),(-0.5,-1.0,0.0),(0.0,0.0,0.0)$ and $(0.0,0.0,0.0)$, respectively. These trajectories have slowed down and have been terminated when $t=600$. The first trajectory uses 74723 calculations while the last four trajectories only use about 7500 each. The adaptive numerical method used to calculate the the trajctories takes larger time steps as the trajectory slows down.

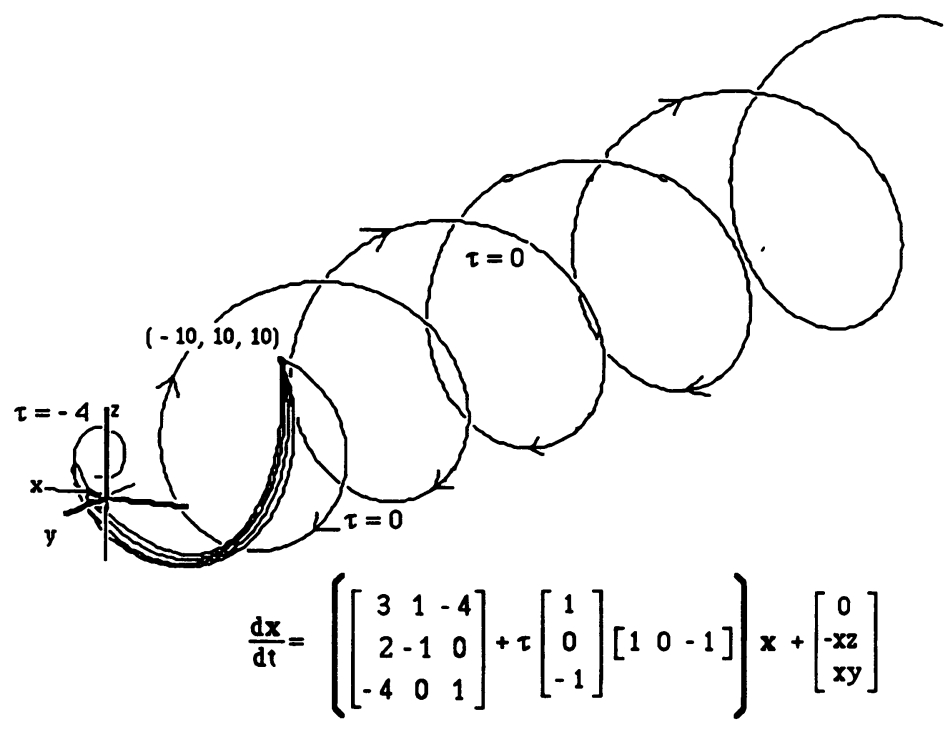

Figure 1:

Theorem 3 Consider the system $M \boldsymbol{x}^{\prime}=N+(A+B K) \boldsymbol{x}+\boldsymbol{f}(\boldsymbol{x})$ with $M, N, A, B$ and $\boldsymbol{f}$ fixed. If $Z_{f}$ contains an $(n-1)$-dimensional subspace of $R^{n}$ and either 
1. $Z_{\boldsymbol{f}} \cap B^{\perp}=\{0\}$ or

2. $Z_{\boldsymbol{f}} \cap B^{\perp} \neq 0$ and $\boldsymbol{u}^{\perp} A \boldsymbol{u}<0$ for all nontrivial $\boldsymbol{u} \in Z_{\boldsymbol{f}} \cap B^{\perp}$

then there exists a matrix $K$ such that the system is point dissipative.

Proof. Under the hypothesis, we would like to show that there exists a negative number $\tau$ such that $\boldsymbol{x}^{T}\left(A+\tau B B^{T}\right) \boldsymbol{x}<0$ for all $\boldsymbol{x} \in Z_{\boldsymbol{f}}$. The result follows from Theorem 1 with $K=\tau B^{T}$.

Case 1. Suppose that $Z_{\boldsymbol{f}} \cap B^{\perp}=\{0\}$. Let $\mathcal{N}=\left\{\boldsymbol{x} \mid \boldsymbol{x} \in Z_{\boldsymbol{f}},\|\boldsymbol{x}\|=1\right\}$. $\mathcal{N}$ is a compact set in $R^{n}$. Since the function $\phi(x)=\frac{x^{T} A \boldsymbol{x}}{\left\|B^{T} \boldsymbol{x}\right\|^{2}}, \boldsymbol{x} \in \mathcal{N}$ is continuous on $\mathcal{N}$ there exists $\boldsymbol{x}_{0} \in \mathcal{N}$ such that $\phi(x) \leq$ $\phi\left(\boldsymbol{x}_{0}\right)=\frac{\boldsymbol{x}_{0}^{T} A \boldsymbol{x}_{0}}{\left\|B^{T} \boldsymbol{x}_{0}\right\|^{2}}$, for all $\boldsymbol{x} \in \mathcal{N}$. Let $\tau$ be a negative number such that $\phi\left(\boldsymbol{x}_{0}\right)+\tau=\frac{\boldsymbol{x}_{0}^{T} A \boldsymbol{x}_{0}}{\left\|B^{T} \boldsymbol{x}_{0}\right\|^{2}}+\tau<0$. For such an $\tau, \phi(\boldsymbol{x})+\tau=\frac{\boldsymbol{x}^{T} A \boldsymbol{x}}{\left\|B^{T} \boldsymbol{x}\right\|^{2}}+\tau \leq \phi\left(\boldsymbol{x}_{0}\right)+\tau<0$ for all $\boldsymbol{x} \in R^{n}$. Now $\boldsymbol{x} \in Z_{\boldsymbol{f}}$ and $\boldsymbol{x} \neq \mathbf{0}$ implies that $\boldsymbol{x}=\|\boldsymbol{x}\| \boldsymbol{u}$, where $\boldsymbol{u} \in \mathcal{N}$. Then $\boldsymbol{x}^{T}\left(A+\tau B B^{T}\right) \boldsymbol{x}=\|\boldsymbol{x}\|^{2}\left[u^{T}\left(A+\tau B B^{T}\right) u\right]<0$ for all $\boldsymbol{x} \in Z_{\boldsymbol{f}}, \boldsymbol{x} \neq \mathbf{0}$.

Case 2. Suppose that $Z_{\boldsymbol{f}} \cap B^{\perp} \neq\{0\}$ and $\boldsymbol{u}^{T} A \boldsymbol{u}<0$ for all nontrival $\boldsymbol{u} \in Z_{\boldsymbol{f}} \cap B^{\perp}$. Let $\mathcal{M}=\left\{\boldsymbol{x} \mid \boldsymbol{x} \in Z_{\boldsymbol{f}} \cap B^{\perp},\|\boldsymbol{x}\|=1\right\}$ and $\mathcal{N}=\left\{\boldsymbol{x} \mid \boldsymbol{x} \in Z_{\boldsymbol{f}} \cap S(B),\|\boldsymbol{x}\|=1\right\}$. By Lemma 5 there is a negative number $\tau$ such that $\boldsymbol{x}^{T}\left(A+\tau B B^{T}\right) \boldsymbol{x}<0$ for nontrival $\boldsymbol{x} \in Z_{\boldsymbol{f}}$.

Example for Theorem 3. Consider the control system $\boldsymbol{x}^{\prime}=A \boldsymbol{x}+\boldsymbol{f}(\boldsymbol{x})+B \boldsymbol{u}$, where

$$
A=\left[\begin{array}{ccc}
-1 & 0 & 4 \\
3 & 1 & 4 \\
2 & -1 & 1
\end{array}\right], \quad \boldsymbol{f}(\boldsymbol{x})=\left[\begin{array}{c}
0 \\
-x z \\
x y
\end{array}\right] \text { and } B=\left[\begin{array}{l}
0 \\
1 \\
1
\end{array}\right]
$$

Note that $A$ is not positive a definite matrix, $\left[\begin{array}{lll}1 & 0 & 0\end{array}\right] A\left[\begin{array}{l}1 \\ 0 \\ 0\end{array}\right]=-1<0$ and $\left[\begin{array}{lll}0 & 1 & 1\end{array}\right] A\left[\begin{array}{l}0 \\ 1 \\ 1\end{array}\right]=5>0$. Here

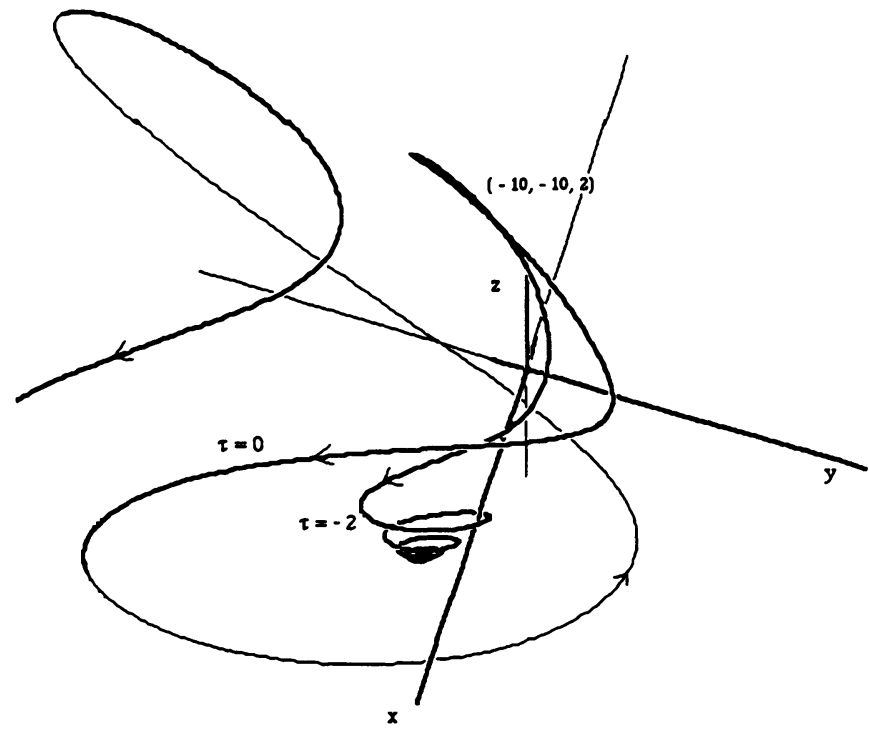

Figure 2: 


$$
B^{\perp}=S\left\{\left[\begin{array}{c}
0 \\
-1 \\
1
\end{array}\right],\left[\begin{array}{l}
0 \\
1 \\
0
\end{array}\right]\right\}
$$

If $\boldsymbol{u} \in B^{\perp}$ then $\boldsymbol{u}=\left[\begin{array}{c}u_{1} \\ u_{2} \\ -u_{2}\end{array}\right], Z_{\boldsymbol{f}}=S\left\{\left[\begin{array}{l}0 \\ 1 \\ 1\end{array}\right],\left[\begin{array}{l}0 \\ 0 \\ 1\end{array}\right]\right\} \cup S\left\{\left[\begin{array}{l}1 \\ 0 \\ 0\end{array}\right]\right\}$. If $\boldsymbol{u} \in Z_{\boldsymbol{f}}$ then either $\boldsymbol{u}=\left[\begin{array}{c}u_{1} \\ 0 \\ 0\end{array}\right]$ or $\boldsymbol{u}=\left[\begin{array}{c}0 \\ u_{2} \\ u_{3}\end{array}\right]$. Thus

$$
Z_{f} \cap B^{\perp}=\left\{\left[\begin{array}{c}
u_{1} \\
0 \\
0
\end{array}\right],\left[\begin{array}{c}
0 \\
-u_{2} \\
u_{2}
\end{array}\right] \mid u_{1} \neq 0, u_{2} \neq 0\right\}
$$

Now $\left[\begin{array}{lll}u_{1} & 0 & 0\end{array}\right] A\left[\begin{array}{c}u_{1} \\ 0 \\ 0\end{array}\right]=-u_{1}^{2}<0$ and $\left[\begin{array}{lll}0 & -u_{2} & u_{2}\end{array}\right] A\left[\begin{array}{c}0 \\ -u_{2} \\ u_{2}\end{array}\right]=u_{2}^{2}-3 u_{2}^{2}+u_{2}^{2}<0$ i.e., if $\boldsymbol{u}$ is a nontrivial element of $Z_{\boldsymbol{f}} \cap B^{\perp}$ then $\boldsymbol{u}^{T} A \boldsymbol{u}<0$. Since $\left[\begin{array}{c}2 \\ -1 \\ 1\end{array}\right]$ is in $Z_{\boldsymbol{f}} \cap B^{\perp}$ and $\left[\begin{array}{lll}2 & -1 & 1\end{array}\right] A\left[\begin{array}{c}2 \\ -1 \\ 1\end{array}\right]=1>0$, Theorem 2 does not apply. Again $\left[\begin{array}{l}0 \\ 1 \\ 1\end{array}\right] \in Z_{\boldsymbol{f}}$ and $\left[\begin{array}{lll}0 & 1 & 1\end{array}\right] A\left[\begin{array}{l}0 \\ 1 \\ 1\end{array}\right]=$ $5>0$ and so Theorem 1 does not apply.

If we choose $k=2 B^{T}$ then

$$
A+B K=A-2 B B^{T}=\left[\begin{array}{ccc}
-1 & 0 & 4 \\
3 & -1 & 2 \\
2 & -3 & -1
\end{array}\right]
$$

The Hermitian part of $A-2 B B^{T}$ is not negative definite, but

$$
\left[\begin{array}{lll}
u_{1} & 0 & 0
\end{array}\right]\left(A+B B^{T}\right)\left[\begin{array}{c}
u_{1} \\
0 \\
0
\end{array}\right]=-u_{1}^{2}<0
$$

and

$$
\left[\begin{array}{lll}
0 & -u_{2} & u_{2}
\end{array}\right]\left(A+B B^{T}\right)\left[\begin{array}{c}
0 \\
-u_{2} \\
u_{2}
\end{array}\right]=-u_{2}^{2}<0
$$

Therefore if $\boldsymbol{u} \in Z_{\boldsymbol{f}}$ then $\boldsymbol{u}^{T}\left(A+B B^{T}\right) \boldsymbol{u}<O$. Hence $M \boldsymbol{x}^{\prime}=\left(A-2 B B^{T}\right)+\boldsymbol{f}(\boldsymbol{x})$ is point dissipative. In Figure 2 two trajectories are given. One when the system is uncontrolled, $\tau=0$, and one when the system is controlled, $\tau=-2$. For both trajectories $(-2,-2,2)$ is the initial point.

\section{Extension of previous results}

The sufficient condition for a quadratic dynamical system to be point dissipative discussed above uses a relation between the quadratic and linear parts of the system when $\boldsymbol{f}$ is conservative. The following lemma allows us to extend the condition to the case where there exists a positive definite matrix $S$ such that $S M=M S$ and $S \boldsymbol{f}$ is conservative. See Bose [5].

Lemma 6 Let

$$
M x^{\prime}=N+A x+f(x)
$$


be a quadratic dynamical system for which there exists a positive definite matrix $S$ such that $S M=$ $M S$ and $S \boldsymbol{f}$ is conservative. Then there exists matrix $H$ for which the change of variables $\boldsymbol{y}=H \boldsymbol{x}$ transforms the dynamical system (1) into $\left(H M H^{-1}\right) \boldsymbol{y}^{\prime}=H N+B \boldsymbol{y}+\boldsymbol{g}(\boldsymbol{y})$ which has a conservative quadratic term. Furthermore, $\boldsymbol{x}^{T} S A \boldsymbol{x}=\boldsymbol{y}^{T} B \boldsymbol{y}$.

When $S f$ is conservative then we say that a vector $\alpha$ is admissible for the dynamical system $M x^{\prime}=N+A x+f(x)$ if $-x^{T} S A x+\alpha^{T} H \boldsymbol{f}(\boldsymbol{x})$ is a positive definite function where $S=H^{T} H$.

When $\boldsymbol{f}$ is conservative then the condition for an admissible $\alpha$ reduces to $-\boldsymbol{x}^{T} A \boldsymbol{x}+\boldsymbol{\alpha}^{T} \boldsymbol{f}(\boldsymbol{x})$ is positive definite. The proofs of the gs when the quadratic part of the system is conservative entail demonstrating the existence of an admissible $\alpha$ for the system. These results can be restated as follows:

Theorem 4 A quadratic dynamical system

$$
M \boldsymbol{x}^{\prime}=N+A \boldsymbol{x}+\boldsymbol{f}(\boldsymbol{x})
$$

is point dissipative when there exists a positive definite matrix $S$ such that $S M=M S$ and $S f$ is conservative and there exists an admissible $\boldsymbol{\alpha}$.

Another direction of generalizing the past results is to consider nonlinear dynamical systems which have nonquadratic nonlinear terms as well as quadratic terms. Relative to the nonlinear terms there again must exist a positive definite matrix $S$ such that the nonlinear terms premultiplied by $S$ are conservative. See Bose [5].

Theorem 5 When there exists a positive definite matrix $S$ such that $S M=M S, S g$ and the quadratic function $S \boldsymbol{f}$ are conservative, and for $S=H^{T} H$

Condition (A) For some admissible $\alpha$ for $M \boldsymbol{x}^{\prime}=N+A \boldsymbol{x}+\boldsymbol{f}(\boldsymbol{x})$ there exists an ordered triple of numbers $(\epsilon, C, m)$ such that $-\boldsymbol{\alpha}^{T} H \boldsymbol{g}(\boldsymbol{x}) \leq C\|\boldsymbol{x}\|^{2-\epsilon}$ for all $\boldsymbol{x}$ with $\|\boldsymbol{x}\| \geq m$.

then $M \boldsymbol{x}^{\prime}=N+A \boldsymbol{x}+\boldsymbol{f}(\boldsymbol{x})+\boldsymbol{g}(\boldsymbol{c})$ is point dissipative.

Note that condition (A) can be replaced by either of the stronger conditions (B) or (C).

Condition (B) There is an admissible $\alpha$ for $M \boldsymbol{x}^{\prime}=N+A \boldsymbol{x}+\boldsymbol{f}(\boldsymbol{x})$ and $\|\boldsymbol{g}\|=o\|\boldsymbol{x}\|^{2}$.

Condition (C) There is an admissible $\alpha$ for $M \boldsymbol{x}^{\prime}=N+A \boldsymbol{x}+\boldsymbol{f}(\boldsymbol{x})$ and $\boldsymbol{g}$ is bounded.

Theorem 6 A quadratic dynamical system $M x^{\prime}=N+A x+f(x), A$ a matrix and $f$ a quadratic function, is point dissipative when

1. there exists a positive definite matrix $S$ such that $S M=M S$ and $S f$ is conservative,

2. the zeros of $f$ contains an $(n-1)$-dimensional subspace, and

3. $z^{T} S A z<0$ for any $z$ which is a nontrivial zero of $f$.

This result can be generalized by adding to the differential equation any conservative function $g(x)$ whose growth is restricted. The corollary states this condition. 
Corollary 1 Let $g$ and the quadratic function $f$ be conservative. If $Z_{\boldsymbol{f}}$ contains an $(n-1)$ dimensional subspace and $\boldsymbol{x} \neq 0$ in $Z_{f}$ implies $\boldsymbol{x}^{T} A \boldsymbol{x}<0$ and

Condition (A) For some admissible $\alpha$ there exists an ordered triple of numbers $(\epsilon, C, m)$ such that $-\boldsymbol{\alpha}^{T} \boldsymbol{g}(\boldsymbol{x}) \leq\|\boldsymbol{x}\|^{2-\epsilon}$ for all $\boldsymbol{x}$ with $\|\boldsymbol{x}\| m$. then $M \boldsymbol{x}^{\prime}=N+A \boldsymbol{x}+\boldsymbol{f}(\boldsymbol{x})+\boldsymbol{g}(\boldsymbol{x})$ is point dissipative.

Note that condition (A) can be replaced by either of the stronger conditions (B) or (C).

Condition (B) There is an admissible $\alpha$ for $M \boldsymbol{x}^{\prime}=N+A \boldsymbol{x}+\boldsymbol{f}(\boldsymbol{x})$ and $\|\boldsymbol{g}\|=o\|\boldsymbol{x}\|^{2}$.

Condition (C) There is an admissible $\alpha$ for $M \boldsymbol{x}^{\prime}=N+A \boldsymbol{x}+\boldsymbol{f}(\boldsymbol{x})$ and $\boldsymbol{g}$ is bounded.

\section{Conclusions}

Quadratic and "almost" quadratic nonlinear systems can exhibit a wide range of qualitative behavior. Even the subclass of such systems with compact attractors contains systems with point attractors, limit cycles and strange attractors, see Sparrow [17]. Linear feedback control problems with system objectives of steering to desired limit points or of minimizing the diameter of a compact attractor have yet to be formulated and solved. This paper represents only a first step, i.e., using linear feedback to produce a controlled system with a compact attractor. An alternate approach might be constructed along the lines of Chow [8] and Kokotovic [13, 12]. Such an approach does not seem to be as closely related to the linear theory as the approach outlined here.

\section{References}

[1] A. K. Bose, A. S. Cover, AND J. A. RENEKE, On point dissipative systems of differential equations with quadratic nonlinearity, Internat. J. Math. and Math. Sci., 14 (1990), pp. 99-110.

[2] - A class of point dissipative n-dimensional nonlinear dynamical systems, Proceedings of the Twenty-fourth Southeastern Symposium on System Theory, (1992), pp. 2-6.

[3] - Sufficient conditions for point dissipative quadratic nonlinear dynamical systems, Proceedings of the Third International Symposium on Differential Equations, Bulgaria, (1992).

[4] - On point dissipative n-dimensional systems of differential equations with quadratic nonlinearity, Internat. J. Math. and Math. Sci., 16 (1993), pp. 139-148.

[5] - Linear feedback control of nonlinear systems with a dominating conservative quadratic term. submitted, 1994.

[6] A. K. Bose AND J. A. RENEKE, Sufficient conditions for two-dimensional point dissipative nonlinear systems, Internat. J. Math. and Math. Sci., 12 (1989), pp. 693-696.

[7] F. Brauer and J. Nohel, Qualitative Theory of Ordinary Differential Equations, W. A. Benjamin, Inc., New York, 1969.

[8] S.-N. CHow, Methods of bifurcation theory, Springer-Verlag, Berlin and New York, 1982.

[9] J. B. CRUz ANd P. V. Koкотоvic, eds., Feedback systems, McGraw-Hill, New York, 1971.

[10] J. GUCKenheimer AND P. Holmes, Nonlinear oscillations, dynamical systems and bifurcations, Springer-Verlag, Berlin and New York, 1983. 
[11] R. A. HoRn And C. R. Johnson, Matrix Analysis, Cambridge University Press, Cambridge, 1985.

[12] P. V. Кокотоvic, ed., Foundations of adaptive control, Springer-Verlag, Berlin and New York, 1991.

[13] P. V. Kokotovic, A. Bensoussan, AND G. Blankenship, eds., Singular perturbations and asymptotic analysis in control, Springer-Verlag, Berlin and New York, 1987.

[14] J. LaSAlle AND S. Lefschetz, Stability by Liapunov's direct method with applications, Academic Press, New York, 1961.

[15] E. LoRenz, Deterministic non-periodic flow, J. Atmos. Sci., 20 (1963), pp. 130-141.

[16] D. L. Russell, Mathematics of Finite-Dimensional Control Systems; Theory and Design, Marcel Dekker, Inc., New York and Basel, 1979.

[17] C. SParrow, The Lorenz equations: Bifurcations, chaos and strange attractors, SpringerVerlag, Berlin and New York, 1982.

[18] S. Wiggins, Global bifurcations and chaos, Springer-Verlag, Berlin and New York, 1988. 


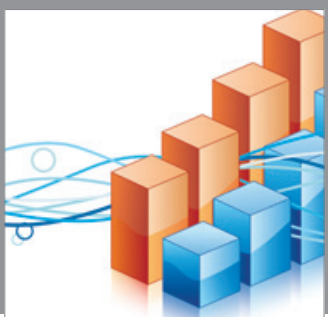

Advances in

Operations Research

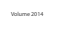

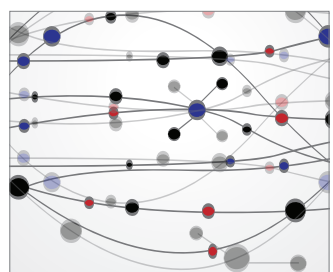

\section{The Scientific} World Journal
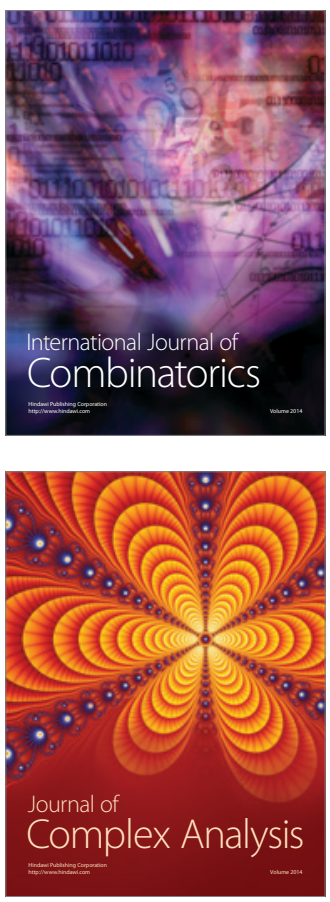

International Journal of

Mathematics and

Mathematical

Sciences
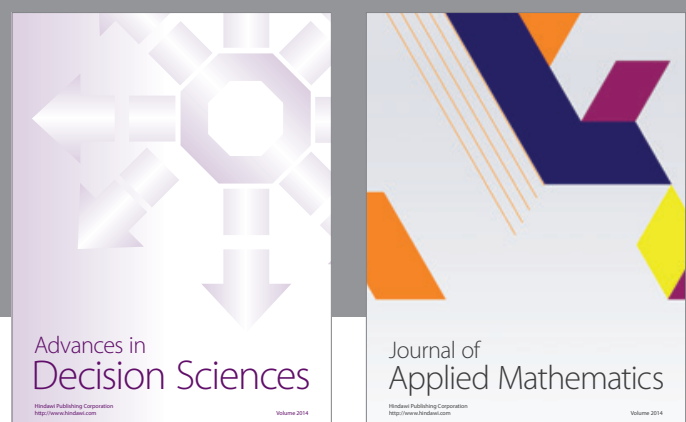

Journal of

Applied Mathematics
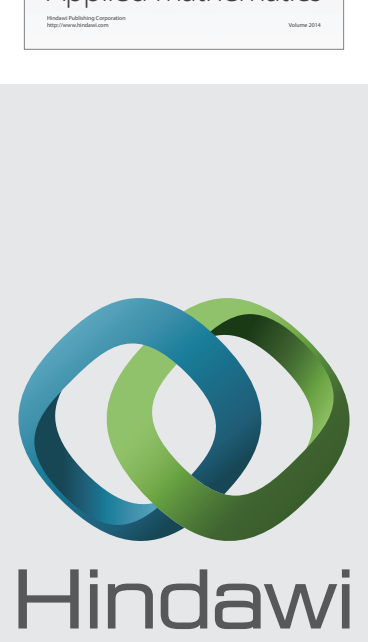

Submit your manuscripts at http://www.hindawi.com
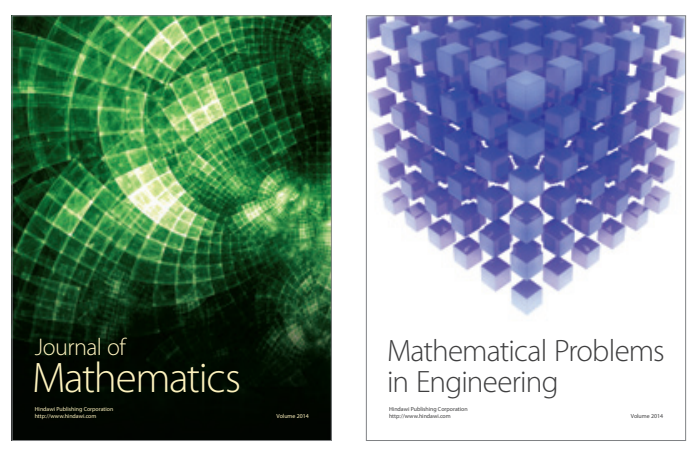

Mathematical Problems in Engineering
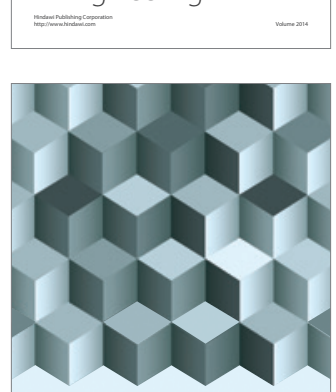

Journal of

Function Spaces
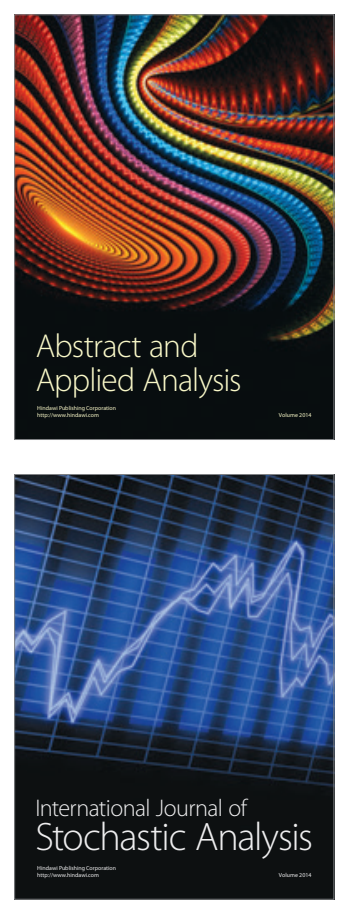

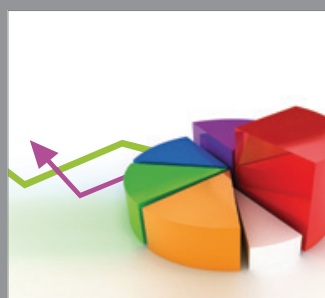

ournal of

Probability and Statistics

Promensencen
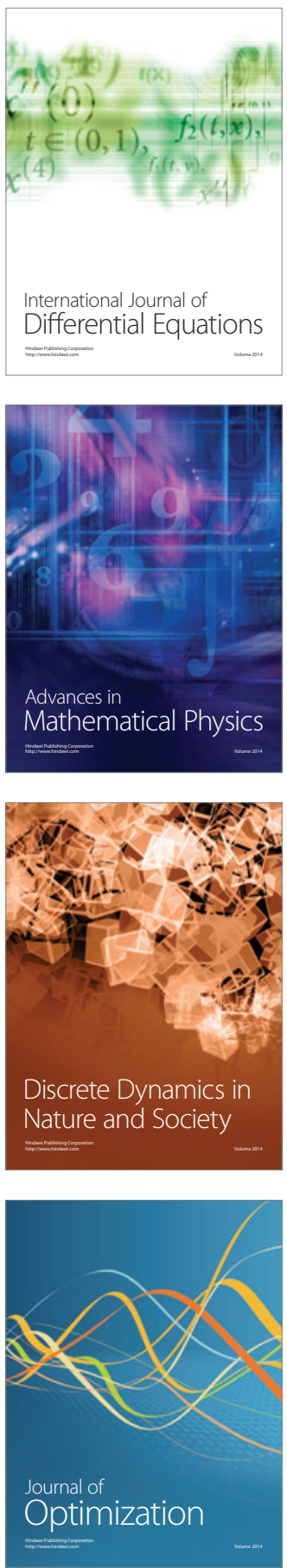